\title{
Networking in the Food Sector of Regional Economy
}

Submitted 19/08/19, 1st revision 20/09/19, 2nd revision 28/10/19, accepted 21/11/19

\author{
Serebryakova N.A. ${ }^{1}$, Sirotkina N.V. ${ }^{2}$, Stukalo O.G. ${ }^{3}$, Dorokhova N.V. ${ }^{4}$
}

\begin{abstract}
:
Purpose: The article aims to study the features and the directions of development of network interaction of subjects of the food sector in different regions of the economy.

Design/Methodology/Approach: The use of different methodological approaches allowed us to formulate a holistic management concept for the development of a network of competences in the food sector of the regional economy.

Findings: The study identified the subjects of such interaction, as well as features that allow them to unite into groups that form networks of competencies. Considering the food sector of the region's economy as a socio-economic system, noted its extreme structural complexity, which necessitates the development of special approaches to its management, or rather the need for structural management is presented. The format of such interaction is a network of competencies, representing a spatially localized socio-economic structure formed on the initiative of active subjects of the regional economy.

Practical Implications: The results obtained can be used as a basis for designing a network form of development of the food sector of the region's economy, taking into account the nature of placement and interaction of participants, as well as allowing for effective use of their resource capabilities, experience and knowledge.

Originality/Value: In order to overcome the problems associated with the formation of network identity, network management competence is proposed to use a structured approach allowing them to make targeted decisions based on objective conditions and institutional nature.
\end{abstract}

Keywords: Regional economy, food sector, networking.

JEL code: $R 59, R 58$.

Paper Type: Research article: Regional Economics, Food Sector.

${ }^{I}$ Professor of the Departmen Theory of economics and accounting policy Voronezh State University of Engineering Technologies, Voronezh, e-mail: nad.serebryakova@mail.ru ${ }^{2}$ Professor of the Department of digital and industry Economics Voronezh state technical University,Voronezh,e-mail: docsnat@yandex.ru ${ }^{3}$ Professor of the Department of management, organization of production and industry Economics Voronezh State University of Engineering Technologies, Voronezh, e-mail: stukalo_oksana@mail.ru

${ }^{4}$ Associate Professor of the Departmen trade and commodity science Voronezh State University of Engineering Technologies, Voronezh, e-mail: nv dorohova@mail.ru 


\section{Introduction}

Spatial and economic transformation of the food sector is manifested in formation of structures combining entities of different sectors of regional economy that have convergent features. At the same time, the processes are observed that correspond to objective laws and lead to a succession of formations, from chaos to hierarchy and to improved conflict-free structures. In this context, elaboration of a network form of development of the food sector of regional economy, which will take into consideration the nature of participants' location and interaction among them and will enable to use their resource capabilities, experience and knowledge efficiently, generates a significant scientific interest. Gnoseological interest to this issue stems from the fact that until now no theoretical constructions, methodological statements, and practical recommendations have been developed that would reveal the prospects of consolidation of a global network resource of competencies and specific aspects of management of network of competencies development in the food sector of the regional economy.

Research into the food sector of regional economy is based on multidisciplinary approaches that presume application of different criteria of classification. General presumption for determination of the food sector of regional economy is the purpose, which is to provide internal (local) and external for the region (national) markets with food products and services connected to production and consumption of food products. Sectorial (technological) and intersectorial (communicational) operations arising in the process of production of food products form the basic structure for different types of relationship, which leads to formation of a special social and economic system - food sector of regional economy - that combines entities of regional economy involved in the processes of identification and scientific justification of the need in food products, design, production and sale of food products. Such system is represented by the aggregate of resources defining capabilities of structural elements and the pattern of their interaction. Such attitude does not contradict with methodological statements of the modern theory of regional development, according to which the regional economy is considered as a system of subject-object relations, the structure of which is formed by material and technical, financial, information, technological, organizational, social and economic links (Khorev et al., 2015; Nagimov et al., 2018).

Formation of the food sector as a social and economic system of meso-economic level is characterized from a position of variance and invariance. Invariant block is formed by:

(1) purposes, which are to provide population of the region with quality food products;

(2) tasks related to implementation of program for production of food products in the region, achievement of targeted indicators in respect of addressing intraregional needs;

(3) functions of entities of the food sector of regional economy as the elements of a social and economic system, which represent types of activities specific to them and aimed at addressing the needs of region's population in food products (Marwa et al., 2017). 
The aforementioned block shall be considered as conditionally invariant because despite the fact that opinions on its content are rather obvious and straightforward, entities of regional economy are characterized by opportunist behavior that disrupts configuration of sustainable and balanced development of the region.

Variable components of the food sector of regional economy cause fluctuations in the processes of its functioning. Variance is defined by location and development, and location comes first since the efficient functioning of the food sector of regional economy more than other sectors depends on the existence of favorable factors for production location, such as environmental conditions and relative costs of production. Being general for the theory of regional economy, substantive fundamentals of location and development are added in this research with specific features connected to perception of the food sector as a spatially localized network of competencies that is forming according to manufacturing traditions, patterns and potential to produce food products from local raw materials with higher productivity in comparison with other regions. Variable block is represented by factors, i.e. particular conditions and reasons, that affect disposition of types of activity and mediate the effect of patterns and principles of productive forces distribution, namely: energetic, ecological, informational, water, labor, raw material, land and transport factors.

While considering the food sector of the regional economy as a social and economic system, it was noted that it is characterized by extreme structural complexity that requires development of special approaches to the management of it, or rather, the structural management. According to modern understanding of mesoeconomic processes, the object of management cannot be separated from the subject of management, as all entities of economy that perform different kinds of activity according to their abilities take part in managerial interaction holding particular status and role position. Network of competencies, which is a spatially localized social and economic structure formed upon the initiative of active entities of regional economy, serves as a pattern of such interaction. In the context of the food sector of regional economy, competencies mean capabilities of its participants to form a new pattern, culture and philosophy of production and consumption of food products, which can contribute to ensuring the high level and quality of living conditions of population (Gumidullaeva, 2018).

The basis for understanding of this definition is a concept of networking and cluster development that reveals the specific aspects of integration under current conditions. These aspects mean that the most competitive participants of economic relations from different sectors, driven by self-interests, enter into interaction based on partnership, parity and free will, but, in doing so, they do not form any legally arranged unions (Serebryakova et al., 2017). Having applied the symbiosis of the analogue method (comparing the subjects of a network of competencies with the subjects of social networks) and the method of morphological analysis, the authors suggest separating seven groups of entities of the food sector of regional economy, whose capabilities and participation form a network of competencies. Differentiated 
according to the type of behavior, the entities of the food sector of the regional economy have been identified using the Russian term and its English translation, so that the first letters of the English definitions made the word "network" (Table 1).

Table 1. Entities of the food sector of regional economy that form the network of competencies

\begin{tabular}{|l|l|}
\hline $\begin{array}{l}\text { Group of } \\
\text { entities }\end{array}$ & Group members \\
\hline $\mathbf{N}$ (newcomer) & $\begin{array}{l}\text { «NEWCOMERS»: organization recycling SHW; organizations providing } \\
\text { transport, logistics, information and telecommunication services; } \\
\text { organizations of the foodservice sector }\end{array}$ \\
\hline $\mathbf{E}$ (enthusiast) & $\begin{array}{l}\text { «ENTHUSIASTS»: processing and agricultural enterprises; wholesale and } \\
\text { retail trade organizations }\end{array}$ \\
\hline $\begin{array}{l}\text { T } \\
\text { (troublemaker) }\end{array}$ & $\begin{array}{l}\text { «TROUBLEMAKERS»: mass media; industrial business papers; } \\
\text { controlling and supervising organizations }\end{array}$ \\
\hline $\mathbf{W}$ (watcher) & $\begin{array}{l}\text { «WATCHERS»: organizations carrying out environmental and social and } \\
\text { economic monitoring; population; organizations carrying out public } \\
\text { expertise }\end{array}$ \\
\hline $\mathbf{O}$ (originator) & $\begin{array}{l}\text { «ORIGINATORS»: organizations of education, research and development } \\
\text { sector; organizations of physical culture and sports sector }\end{array}$ \\
\hline $\mathbf{R}$ (redactor) & $\begin{array}{l}\text { «REDACTORS»: executive authorities; population; civil society } \\
\text { institutions }\end{array}$ \\
\hline $\mathbf{K}$ (keen) & $\begin{array}{l}\text { «KEEN»: population; enterprises and organizations showing interest in } \\
\text { development of the food sector and enthusiastically contributing to it }\end{array}$ \\
\hline
\end{tabular}

Opinions on structural identity of the entities of the food sector of regional economy have been formed by the authors by means of separating extended groups (types) as it is impossible to take into consideration different individual characteristics of entities of interaction. Primary (inherent (immanent) characteristics) and secondary (obtained in the process of interaction) characteristics of structural identity have been used as a criterion for separating groups shown in Table 1. In order to carry out detailed analysis, secondary characteristics were divided into permanent (invariable) and variable, i.e. changeable under the influence of external conditions and endogenous factors.

An important feature of networking among the entities of the food sector of regional economy is the absence of hierarchy, which is the fundamental difference between the network and the system. Scaling up of the network nodes makes it possible to see that they are just smaller networks, since in economic networked world there are no hierarchies; there are only networks that are inbuilt in other networks. Network structure is the combination of two possible types of elementary networks - close circuit and open circuit, as well as a mixed-type circuit. Mentioned theses resolve the contradiction enabling to balance and harmonize interaction among the entities of the food sector of regional economy: absence of hierarchy eliminates the need for certain entities to dominate and makes it possible to avoid conflicts connected to it. Emergence of network structures (networking) in sub-systems of regional social and 
economic system is the result of finding by particular actors of required resources, the bearers of which are other participants of interaction. In this context, the network of competencies forms as a result of search for and finding of opportunities to solve resource problems. Entities of regional economy feel the need in lacking competencies, that is, the search for opportunities comes first and followed by establishment of partnership links with the bearers of opportunities, i.e., with the entities that have necessary competencies. Competencies are characteristics of entities of the food sector of regional economy, dynamic capabilities that define the potential of the economic entity in the field of integration, creation and reconfiguration of internal and external capabilities to meet the requirements of fastchanging environment.

Network of competencies in the food sector of economy looks like a matrix. Planes of interaction among the groups of entities form the following axes: territorial localization, specialization, competition. The space formed by the axes enables to separate three aspects of networks of competencies: substantive, institutional, quasicorporate.

Substantive aspect of interaction among the entities of the food sector of regional economy reveals the pattern of forming a network of competencies in the plane "territorial localization-specialization". On a meso-level, objectification of interaction with due account for territorial localization and specialization connected to it is caused by technological particularities of production cycle, which is especially evident in the food sector of the regional economy. This research proves that transport factor is no longer relevant and it is not a decisive factor for making a decision concerning disposition of production as it was in the times of Thunen and Launhardt. In the current context, territorial localization of the bearers of competencies forms subject to concentration of specialized resource capabilities, which is manifested in formation of clusters and general differentiation of the economic space of a region (Serebryakova et al., 2016a).

Formation of clusters (engineering and manufacturing cross-section) and fundamental differences in social and economic condition of municipal entities (territorial cross-section) is the result of one problem, to be more precise, of the behavior of meso-economic pattern according to which entities of regional economy turn out to be the bearers of different competencies and compete with each other for possibility to use them with the maximum benefit for themselves. This circumstance highlights the need to separate institutional aspects of interaction among the entities of the food sector of regional economy and reveals the pattern of network of competencies formation in the plane "specialization - competition".

Identification of the institutional aspect is also connected to one more objective law - competitive methods aimed at expansion of market by means of removing other agents are less effective than methods of developing "competitive collaboration" 
that lays groundwork for realization of participants' opportunities by means of joint solving of their problems.

Quasi-corporate aspects of interaction among the entities of the food sector of economy reveal the pattern of the network of competencies formation in the plane 'territorial localization - competition', which is connected to the existence of the following objective law: driven by the desire to improve their competitive abilities, entities of the regional economy enter into interaction without institutionalization motivated by their desire to cooperate (not to compete) with organizations located in the territory of the region that have competencies they are lacking in. Due to that, it is worth mentioning that the nature and the content of relationships arising among the subjects of the network are defined with consideration of dualism of interests shown by them.

Potential opportunity for networking lies in the structure of entities of the food sector of regional economy, which is represented by all possible groups defined in accordance with the authors' approach NETWORK, but mainly by organizations that form the group of enthusiasts. Having examined the situation currently existing in the Voronezh region, it was established that generally, only the half of production capacities of enthusiasts, as the most probable participants of the network of competencies, is used. It means that the potential of this group, their resource capabilities shaping competencies are not realized.

At the same time, there is a trend towards increasing the use of production capacities in those fields of activity that have been boosted by introduction of sanctions against our country: meat and edible offal of meat-producing animals, meat and edible offal of poultry, soft drinks, butter and butter paste, cheese and cheese products. The example of using production capacities uncovers the problem of searching a partner capable to realize its potential and fill the need in cooperation. It also proves the potential of networking development with the view of solving the following tasks:

1) to balance resource capabilities, restrictions and needs;

2) to improve quality of the living environment within agglomerations;

3) to trigger cross-sectorial interaction that forms institutional basis for development of the food sector and regional economy in general.

Separation of substantive, institutional and quasi-corporate aspects of forming network of competencies evidences that structural links emerging in the process of networking cover almost all fields of regional economy activity and form the structural frame of the economic space of the region, sustainability of which is ensured by participants' interest in partnership based on the belief that the competitive cooperation offers much more advantages and opportunities than competitive battle. 


\section{Methods}

This research represents methodology of development of networking among the entities of the food sector of regional economy in general perspective. It gradually reveals philosophical, cognitive and technological aspects. In this research, philosophical aspect of methodology in the field under consideration means the aggregate of methods that enable to synthesize theoretical opinions on the issue of network of competencies development management as a way to regulate and activate opportunities, resources and knowledge of the entities of the food sector of regional economy showing interest in its development and demonstrating responsible behavior with due consideration of their position, which is defied from the position of "role-status".

As part of development of philosophical aspects of methodology in accordance with the principle of ontological engineering, the new economic category has been synthesized in this research -network of competencies of the food sector of regional economy. It is shown as resources, resource capabilities, experience and knowledge necessary to produce quality food products in the amount and in the assortment satisfying the needs of population of the region provided by the dynamic set of interconnected economic agents (population, executive authorities, agricultural and processing organizations, organizations in the field of education, research and development, medicine and physical culture, organizations recycling solid household waste).

Application of competencies by the subjects of network differentiated into groups according to their role and status has a cyclical pattern. Thus, the structure of the network is reproduced. Some of competencies are acquired by the subjects of network at the stage of their involvement in its structure. The main features of the network are: absence of formal restrictions in respect of number and content of participants; existence of endogenous information stratification; endogenous (internal to the structure of the network) identity; conformity of the network's participants' behavior (Serebryakova et al., 2016b; Marwa et al., 2017).

Resources of a network of competencies mean qualities that promote improvement of efficient functioning of a network as a structure. Owing resources defining their competencies, participants of the network enter into interaction that promotes formation of joint competencies. Development of joint competencies, in terms of its functional content, is also a resource.

Seeing the complexity of networking, we suggest considering network of competencies from the following positions: subject, i.e., a participant from among the entities of the food sector of regional economy; subject matter-a technological operation that requires application of resources, knowledge and experience, i.e., use of competencies, in order to be performed; project-in the context of implementation of a project or results-based approach to the management of regional economy. 
Dominant characteristics of the network of competencies considered from the position of subject, subject matter and project, are:

(1) each participant of the network has certain functional competency (set of competencies) that are mostly localized;

(2) the number of participants of the network is rather high, however, it is comparable to the number of technological stages of co-creation cycle implemented by participants of the network and defined by its subject content, and is incomparably less than the number of projects implemented by the participants of the network;

(3) participants of the network are autonomously functioning organizations that are engaged in interaction due to their desire for mutual satisfaction of their resource needs and their interest to realize their resource capabilities;

(4) in terms of project implementation, the role of one participant is insignificant in comparison with the value of participants' interest in cooperation and their mutual responsibility;

(5) integral characteristics of a network as a holistic structure are of great importance, so are the characteristics of individual participants;

(6) absence of hierarchy in the network does not allow considering it as a system, that is why an integral characteristic of the network is its additivity.

Philosophical opinions on the methodology of a network of competencies formation are added in this research with the following imperatives:

1. Formation of a network of competencies is always connected to the location of entities of regional economy. Indeed, the problem of networking is the problem of location. Similar to expansion of network companies in the regions with high concentration of consumer's demand, formation of a network of competencies is noticed in the regions with a high concentration of participants co-creating value but positively competing with each other, since the absence of competition deprives entities of regional economy of the opportunity and desire to develop.

2. Basically, network of competencies is a cluster with the institutional agent, where the state acts as such. Seeing new endogenous and exogenous challenges, the task of developing and implementing of state economic policy involves regulation of interaction among the entities of regional economy in the directions that limit the regional growth. It is possible to solve this problem if the state will be lodged with the function of the institutional agent performing initiative responsible and creative activity in the system of large risks and uncertainty, which is aimed at formation of favorable conditions for fair competition.

3. Networks of competencies have regional specialization. The need to separate regional networks of competencies stems from the existence of a federal level of the management of economy, whose potential shall not be doubled at the regional level. 
4. Executive authorities perform the function of providers of fair competition at the regional market, because they regulate allocation of resources among the regional networks of competencies and federal networks in the region. Tools that allow executive authorities to keep the balance of competencies, promote completion of lacking competencies, redistribute resources and regulate realization of resource capabilities are project management and the system of state programs. It is not yet possible to evaluate the efficiency of the project management due to the lack of representative selection of data displaying its results. State programs designed and adopted for practical implementation in the Russian regions are estimated by the authors as ambiguous. However, in general, there is a space for improvement.

5. Criterion for formation and development of the network is fairness, for everything that limits opportunities is unfair. At the level of regional economy, the issue of fairness is directly connected to the allocation of funds from the Federal budget, inter-budgetary relations, implementation of state programs and application of other tools of state regulation. Economic space of our country is characterized by significant differentiation of regions in terms of the level of economic development, which is by itself, an extremely negative factor. However, recently some trends have been noticed that significantly worsen the situation and that are the signs of "unfairness". Thus, those regions that have traditionally been the donors of the state budget now turn out to be in the group of subsidized regions, which is the result of inefficient state policy. In our opinion, regional policy shall be aimed at support of donor regions in order to establish conditions for realization of their potential. Unfair reallocation of budgetary funds leads to the decrease in national level of economic development and does not promote formation of competitive environment, which is the basis for networking. Coming back to the functions of the state, it should be noted that executive authorities shall act as an institutional agent that creates conditions for fair competition promoting the development of integration, cooperation and collaboration among the entities of regional economy.

6. Coordination in the network of competencies is ensured by means of formation of internal information space that organizes the flows of innovative knowledge. Rampup of circulating information volumes promoting diffusion of innovations leads to creation of collective objects of intellectual property and improvement of external effect from network's activity.

7. Optimality of the network's structure is defined by: 1) economic efficiency of its functioning; 2) the speed of knowledge generation; 3) the speed of accumulating useful experience; 4) the speed of finding lacking resources. Historical facts proving the reasonableness of these theses can be found when analyzing new economic policy as the most successful reform ever been implemented in our country during the last 100 years. Productivity of NEP was mainly due to practical application in managerial practice of mechanisms of activation of parity cooperation in the form of free-will partnership aimed at mutual satisfaction of needs and realization of own 
opportunities with the maximum output, i.e. in accordance with the authors' opinions on the networks of competencies development.

8. Stability of the network is ensured by means of preserving independence and invariance of partners in the process of realization of their competencies, because joint activity results in convergence and further unification of competencies. Absence of unique competencies deprives participants of the network of attractiveness, does not allow the competitive environment to form and inevitably leads to destruction of a network, thus creating vacuum in economic space of the region, which is unacceptable in case of the food sector of regional economy since interruptions in its functioning leads to the loss of food and economic safety of the country.

9. Effective motivating tool for participants of the network is formation of the need in cross-functional cooperation on the terms of parity and equality, because by neutralizing the priority of one participant of the network over the others it is possible to guarantee protection against opportunist behavior.

Cognitive aspects of the methodology of network of competencies development management in the food sector of regional economy are considered in this thesis with due consideration of dialectic of crises that go on in this sector and, in general, in national and international economy:

1. Development of the food sector of regional economy is affected by: (a) world trend and nature of its connection with national economy; (b) context history of development of a food sub-system of national social and economic system; (c) crises caused by technological, natural and climate, geopolitical and other factors internal to the food sector of regional economy.

2. Economic crises affecting the food sector of regional economy cause "creative destruction".

3. Coincident point of the downward phase of a crisis cycle, that has been observed as a result of overlapping of different crises, every time turns out to be on the level above the previous one, which corresponds to general incremental positive trend of economy development.

4. Participants of networking form integrated community by overcoming crisis situations of meso- and macro economical level.

5. Conflicts accompanying crisis trigger positive development of networks and act as catalysts. Opportunism and procrastination shown by the participants act as inhibitors in the process of network development management.

Cyclical nature of economic development proved in different times by scientists in our country and abroad is a reason to think that growing phase and sustainable development come after recession and crisis. Thus, by 2020 the downward phase of the cycle will have been over and the entities of regional economy will be able to 
function at a totally new level. They will start from the positions that are above the bottom of the previous crisis and will accumulate the bundle of system innovations.

Overlapping of different crises and coincidence of their downward phases in time is the period for activation of managerial impacts from the state, i.e., the time for development and implementation of specific approaches to state regulation. Analysis of national and foreign expertise shows that subject to efficient use of mechanisms of state regulation, executive authorities can modify the path of cycle development, which means to reduce or expand its duration, enlarge the amplitude etc. This especially concerns medium-term cycles. Investments are an effective tool of anticrisis management. As a result of investment support for investment projects significant for certain segments of regional economy, medium-term cycles acquire the most acceptable $\mathrm{V}$-shaped configuration, which is characterized by reduction of duration of a downward phase.

Table 2. Interconnection of philosophical, cognitive and technological aspects of the methodology of network of competencies development management in the food sector of regional economy

\begin{tabular}{|c|c|c|}
\hline $\begin{array}{l}\text { Philosophical } \\
\text { aspects }\end{array}$ & Cognitive aspects & Technological aspects \\
\hline $\begin{array}{l}\text { Formation of the } \\
\text { network of } \\
\text { competencies is } \\
\text { performed } \\
\text { according to } \\
\text { location of entities } \\
\text { of regional } \\
\text { economy }\end{array}$ & $\begin{array}{l}\text { Food sector of regional economy } \\
\text { develops under the influence of } \\
\text { factors that define the world trends } \\
\text { of economic development and the } \\
\text { nature of its interconnection with } \\
\text { national and regional economy. }\end{array}$ & $\begin{array}{l}\text { Formation and development of the } \\
\text { network of competencies shall be } \\
\text { carried out in accordance with a } \\
\text { methodical approach that reveals } \\
\text { prospects of cluster and network } \\
\text { interaction among the entities of } \\
\text { the food sector of regional } \\
\text { economy }\end{array}$ \\
\hline
\end{tabular}

Conclusion: development of the network of competencies is performed under the influence of endogenous (location of entities of the food sector of regional economy) and exogenous (cyclical nature of development of regional, national and international economy) factors and requires regulation by means of coordinating managerial interaction among the executive authorities and other entities of the food sector of regional economy, which is aimed at formation and development of the network of competencies.

\begin{tabular}{l|l|l}
$\begin{array}{l}\text { Basically, } \\
\text { network of } \\
\text { competencies } \\
\text { is a cluster with } \\
\text { the institutional } \\
\text { agent, where } \\
\text { the state acts as } \\
\text { such }\end{array}$ & $\begin{array}{l}\text { Development of the food } \\
\text { sector happens as a result of } \\
\text { by cyclically-repeated } \\
\text { economic crises. }\end{array}$ & $\begin{array}{l}\text { The level of networking shall be evaluated } \\
\text { in accordance with the modular system of } \\
\text { evaluation that enables to define the } \\
\text { strength of interaction among the } \\
\text { participants of the network and ensure } \\
\text { selection of the most efficient network } \\
\text { links necessary for development of the } \\
\text { food sector of regional economy. }\end{array}$
\end{tabular}

Conclusion: network of competencies development management is a regulatory activity of executive authorities who act as an institutional agent creating favorable conditions for fair competition among the participants of interaction. Functioning of the participants follows the objective laws, according to which crises inevitably happen and accompany the change of upward and downward phases of economic cycle and lead to the shifts and increase in the 
general level of development of the subjects of interaction.

Networks of $\quad$ Development of entities of the food competencies have regional specialization

sector of regional economy is

characterized by gradual improvement of social and economic

condition of the sector, regional

economy and national economy in

general.

Conclusion: development management of the network of competencies in the food sector of regional economy is reasonable to the extent of competencies and powers of executive authorities to design and implement structural policy that takes into consideration a competitive profile of the region formed in accordance with its territorial particularities and established specialization influencing the emergence of cluster and network structures. Dichotomy of the networking among the entities of the food sector of regional economy displays itself in the fact that on the one hand networks of competencies are the result of formation of regional identity in the fields of specialization and competition of territorially-localized entities; on the other hand - existence of specialized competitive types of activity is a necessary condition for formation and development of the network of competencies.

\begin{tabular}{l|l|l|}
$\begin{array}{l}\text { Executive authorities act as a } \\
\text { provider of fair competition } \\
\text { during the process of } \\
\text { networking. }\end{array}$ & $\begin{array}{l}\text { Development of the food } \\
\text { sector of regional } \\
\text { economy complies with } \\
\text { the particularities of } \\
\text { integral community } \\
\text { formation. }\end{array}$ & $\begin{array}{l}\text { The pattern of regulating the } \\
\text { food sector of regional economy } \\
\text { with due consideration of its } \\
\text { competencies is a project office. }\end{array}$ \\
\hline
\end{tabular}

Conclusion: network of competencies development management is structural because it is performed with consideration of subject-object relations emerging among the participants of the food sector of regional economy in respect of implementation of joint projects that are subject to regulation in accordance with conceptual provisions of project management by means of implementation of respective methodological tools.

\begin{tabular}{|c|c|c|}
\hline & \multirow{3}{*}{$\begin{array}{l}\text { Conflicts arising } \\
\text { out of interaction } \\
\text { among the entities } \\
\text { of the food sector } \\
\text { of regional } \\
\text { economy and } \\
\text { accompanying } \\
\text { crises are the } \\
\text { catalysts of } \\
\text { positive } \\
\text { development. }\end{array}$} & \multirow{3}{*}{$\begin{array}{l}\text { Effective tool of implementing } \\
\text { policy of structural development } \\
\text { management of the food sector of } \\
\text { regional economy is elaboration } \\
\text { of object-oriented and project- } \\
\text { oriented models of networks of } \\
\text { competencies that enable to } \\
\text { separate optimum set of } \\
\text { competencies of the main } \\
\text { participants of the food market. }\end{array}$} \\
\hline & & \\
\hline & & \\
\hline
\end{tabular}

Conclusion: the model of the network of competencies development is a virtual enterprise which shall be managed with the use of advance achievements in the field of management oriented to the micro level of economy and the management that reveals the particularities of managerial interaction on meso- and macroeconomic levels. Functions of institutional support of formation of the network of competencies shall be vested in regional corporation of development. 

During the downward phase of medium-term cycles, the discrete nature of innovations transforming into the form of system and basic ones displays itself. This pattern is of great importance to the development of the food sector of regional economy, regional economy in general and national economy, since due to the activation of networking ensuring cumulative distribution of business activity among the participants of the network it becomes possible to ensure implementation of investment projects promoting the development of the food sector as a sub-system of regional economy and resulting in achievement of multiplying effect.

Block of questions linking together all three aspects of the methodology and drawing attention to technological aspect is the development of principles related to the network of competencies development management in the food sector of regional economy. The following principles have been highlighted during research: the principle of activity; commensurability of changes; structuredness; synchronization; harmonization; mutual usefulness; coherence of changes.

Separation of technological, cognitive and philosophical aspects of methodology, corresponding to the authors' opinion on it, requires coordination (Table 2) in order to achieve the goal of methodology development, which is to define the approach to formation of theoretical views on the problem of the network of competencies development management in the food sector of regional economy. In our opinion, methodology, as a way of learning, is general and cannot be defined through the particular and shall be considered as belonging to the system of higher level. Nevertheless, when developing the methodology, we provide the line of dialectic arguments from the general to the particular and vice-versa.

\section{Discussion}

Food sector of regional economy includes the whole aggregate of entities located in the territory of the region directly producing or supporting production of food products at scales necessary to satisfy the need of the regional market in competitive products and services related to their consumption. The main prerequisite for formation of the food sector as a field that coordinates reasonable activity of entities of regional economy from different sectors is an integration interaction emerging in response to the need to optimize the provision of processing production with national raw materials and establishment of partnerships among innovative, scientific and research, educational and other organizations of the region.

Having examined the origin of different forms of integrated interaction, it was established that the vector of evolutionary development is aimed at complication of a structure and simplification of interaction organization by means of full or partial refusal of hierarchical bureaucratic legally registered relations in favor of initiative partnership, provided, though, that there is a state regulation aimed at provision of fair competition by means of efficient interaction of executive authorities, population, civil society institutions and other providers of regional development. 
Having performed the worldview revolution, having stepped beyond the frames of the transaction costs theory, the modern forms of integration interaction show quasicorporate (not quasi-integration) features. This is mainly because they are characterized by structuredness, common goal orientation of entities involved in interaction. At the same time, they do not involve fixation of ownership relations and operate driven by their own "egoism" that triggers initiation and establishment of partnership links, mainly for implementation of individual projects. Forms of such interaction (development of integration) are clusters and networks of competencies (clusters with an institutional agent). Tools for organization of integration interaction in the forms that promote its development are: outsourcing, which envisages creation of special structures inside the network that provide specialized services to the participants of interaction and external parties; insourcing allowing the specializing structure inside the network to perform a part of functions on a regular or contractual basis, ensure growth of functioning efficiency of the network in general, organize commercialization of the joint competitive advantage created by the participants of the network.

Network structures are the most acceptable form of development of interaction among the entities of the food sector of regional economy due to the large number of direct participants of the production process, processing and distribution of the food products, as well as support and institutional and infrastructural provision.

The concept of the structural development management of the network of competencies is the aggregate of the authors' views on the problem of mutual satisfaction of resource needs of the entities of the food sector of regional economy with consideration of their endogenous and exogenous abilities. Having summarized and systematized theoretical approaches to definition of competencies, the authors have noticed a bifurcation moment in studying of this phenomenon that happened at the beginning of the current century and was caused by transformation of attitude towards strategic management. Transformation showed itself in the development of a modular approach to development of strategies, i.e. possibility to modify them under the influence of dynamically-happening changes that quite often are triggered by the entities of economic interaction themselves in order to avoid stagnation, because stability is not any longer a normal feature of economic relationships aimed, first of all, at satisfaction of customer.

The reasons why the focus of a strategic management has been transferred in the plane of "short-term competitive advantages" are: development of digital economy, expansion of globalization scales; reduction of market barriers, blurring of the sectors boundaries, and nondeterministic nature of consumers and competitors' behavior. Seeing the circumstances given above, a thesaurus of the concept of network of competencies development in the food sector of regional economy has been formed: 
1) authors' understanding of competencies (informational - processing of big data characterizing market, corporate and net (hybrid) transactions; technological support of production and consumption of quality food products in accordance with the concept of healthy eating as a philosophy for the increase of the level and quality of population's living standards; communicative - ensuring of solidarity among the entities involved in interaction by means of the increase of its attractiveness) and network of competencies;

2) definition of a relevant set of resources necessary for development of the network of competencies, and resource capabilities (administrative, arising due to coordination of activity of executive authorities of municipal, regional and federal level; distributable, forming as a result of managerial interaction among the entities of the food sector of regional economy in accordance with institutional conditions defined by existing structural policy aimed at improvement of quality of living standards of population of the region; territorial, connected to the use of the space of a region as a resource element);

3) problems of network of competencies development (problems related to choosing partners among which there is a competition for provision of their competencies and identification of partners as bearers of competencies that the entities of the network of competencies lacking; complexity of distributing tasks among the bearers of competencies and coordination of process of achievement of interaction goals; asymmetry of information support and risk of capture of strategic information, which threatens to continuity of interaction);

4) formation of a network identity (involvement and 'socialization' of participants under the influence of individual (of each subject of interaction) and network-wide identity; institutionalization of networking among the entities of the food sector of regional economy with due consideration of each participant's competencies; identification of functioning principles of the network of competencies with fair competition, the main provider of which is executive authorities implementing the respective policy);

5) specific aspects of structural development management of the network of competencies - organizations of the food sector of regional economy act as a main element of the structure. At the same time, they are subjects and objects of economic relationships emerging in the process of networking which is constructed around the network's nodes "status-role" formed by intersection of competencies in the following planes: "territorial localization - specialization", "specialization competition", "competition - territorial localization". The tool of structural management is formation of patterns of interaction among the subjects of a network. Being the bearers of competencies, the entities of the food sector of regional economy can form the model of filling the economic space of the region taking into account transformed opinion on existing archetypes of entities of regional economy and their interaction as formalized analogue of virtual communication of actors. (Serebryakova et al., 2015)

Food sector of regional economy is a field whose results are the products manufactured by the subjects of integration interaction who are the bearers of 
competencies. Development management of the network of competencies in the food sector of regional economy shall, in our opinion, be aimed at achievement of additivity from interaction following implementation of a convergence principle, i.e. sustainable and goal-oriented development by means of harmonization of plans and projects of participants.

The problem of managing the network of competencies development is complicated by the fact that participants of networking fail to realize that they are actually involved in network structures, since they suffer from motivational skepticism. (Serebryakova, 2016c). This circumstance is one of the endogenous factors negatively influencing the prospects of development management of network of competencies. Other similar factors include: heterogeneity of sectorial membership of network participants and types of activity conducted by them; existence of participants that are significantly different in terms of size and scale of their activity; differentiation of participants in terms of type of ownership and assets structure; significant differences in traditions of informatization of internal and external transactions; influence of "time factor" that shows itself in nonconcurrence of time of involvement into interaction (often accompanied by investment) and making profit.

\section{Conclusion}

Summarizing what has been said above, it should be noted that actual and potentially possible availability of resources directly affects formation of conditions of institutional nature, which define prospects of the network of competencies development in the region and management of it. In our opinion, development of the network of competencies in the food sector is possible in the regions with the following specialization: agricultural, agro-industrial, diversified. Seeing the attempts to ensure transition of our country's economy to the sixth technological paradigm, prerequisites for development of the network of competencies in the food sector may be created also (and even more) in the regions where the residents are the organizations producing high-tech products.

The concept of structural development management of the network of competencies is determined by the following provisions:

1. Values, norms and determinants of fair competition define the conditions for development management of a network of competencies. Such conditions shall be differentiated as being of objective (geo-economical and geo-political location; risks of globalization and transition to the new technological pattern) or institutional (actual availability of resources and prospects of its development; system of intellectual capital of the region) nature.

2. Procedure of a network of competencies development management in the food sector of regional economy includes the following stages: (1) analysis and 
evaluation of challenges and problems faced by regional economy that define the necessity to develop the network of competencies in the food sector; (2) clarification of goals; (3) development of policy and strategy; (4) development and implementation of organizational and management innovations and integration mechanisms; (5) correction of managerial interaction; (6) assessment of efficiency of managerial interaction; (7) verification of results of achievement of structural policy goals.

3. Space of interaction among the entities of the food sector of regional economy differentiated according to their status and role in the process of production of food products and services related to them is three-dimensional and formed by the following axes: territorial localization, specialization, competition. The plane formed by intersection of axes "territorial localization" and "specialization" allows characterizing the network of competencies from the subject's position; plane "territorial localization - competition" - from the position of subject matter; plane "competition - specialization" - from the position of project management.

4. Groups of entities of the food sector of regional economy (NETWORK) that are the bearers of the resource capabilities, knowledge and experience form the network structure by means of the following patterns: information support of interaction (information on the needs of entities of the food sector of regional economy; technological opportunities; lacking competencies; current state and achievement of long-term goals); scientific and methodological support of development of the food sector of regional economy; methodological tools of the network of competencies development used by executive authorities.

Thus, the whole complex of authors' views on the problem of the development management of the network of competencies in the food sector of regional economy has been summarized in the relevant concept that is notable for seeing a network of competencies in the food sector of regional economy as a territorially localized structure involving the bearers and consumers of competencies necessary for its development. In order to avoid problems related to shaping of a network identity, it is suggested that the structural approach shall be used for managing a network of competencies, which will enable to make tailored decisions that take into account conditions of objective and institutional nature.

\section{References:}

Gamidullaeva, L. 2018. Towards combining the innovation ecosystem concept with intermediary approach to regional innovation development. International Journal of Economics and Business Administration, 6(1), 39-53.

Khorev, A.I., Salikov, Y.A., Serebryakova, N.A. 2015. Conceptual features of the balanced development of business organizations. Asian Social Science, 11(20), 22-28.

Marwa, T., Bashir, A., Azwardi, M.A., Thamrin, H.M.K. 2017. Market Integration of Agricultural Products. International Journal of Economics and Business Administration, 5(2), 69-82. 
Nagimov, A.R., Akhmetshin, E.M., Slanov, V.P., Shpakova, R.N., Solomonov, M.P. and Il'yaschenko, D.P. 2018. Foresight technologies in the formation of a sustainable regional development strategy. European Research Studies Journal, 21(2), 741-752.

Serebryakova, N.A., Dorokhova, N.V., Isaenko, M.I.. 2017. Formation of the system of clustering as a means of perspective development of innovational infrastructure of region. Contributions to Economics, 133-142.

Serebryakova, N.A., Salikov, Y.A., Kolomytseva, O.Y., Pakhomova, T.A., Grishchenko, N.V. 2015. Actual issues of planning of well-balanced development of innovative \& investment activities. Asian Social Science, 11(20), 193-205.

Serebryakova, N.A., Semenenko, S.V., Grishchenko, N.V. 2016a. Methodological ways of formation of corporate entities in food industry. Journal of Applied Economic Sciences, 4(11), 616-623.

Serebryakova, N.A., Semenenko, S.V., Grishchenko, N.V., Ulchenko, T.Y. 2016 b.

Competitive Potential of Trade Organization: Theoretical and Methodological Foundations of Formation and Realization. European Research Studies Journal, 2(19), 3-11.

Serebryakova, N.A., Volkova, S.A., Volkova, T.A., Semenenko, S.V. 2016c. Methodological approaches to evaluation of economic security of enterprise. Journal of Applied Economic Sciences, 1(11), 325-335. 